\title{
Role of 18F-FDG PET-CT in Vasculitis: A Prospective Study
}

\author{
Kinga Diana Siczek ${ }^{1}$, Paola Faggioli ${ }^{2}$, Emanuela Venegoni ${ }^{3}$, Luigia Florimonte ${ }^{4}$, \\ Marco Carletto ${ }^{5}$, Fabio Cammelli ${ }^{6}$, Cristina Songini ${ }^{7}$, Alberto Vignati ${ }^{8}$, Rossella \\ Stoico ${ }^{9}$, Antonino Mazzone ${ }^{10}$ and Lorenzo S. Maffioli ${ }^{11}$ \\ 1,5,6,7,8,9,11 PET center, Nuclear Medicine Dept., Ospedale Civile di Legnano, Legnano, Italy \\ 2,3,10 Internal Medicine Dept., Ospedale Civile di Legnano, Legnano, Italy \\ ${ }^{4}$ PET center, Nuclear Medicine Dept., Fondazione IRCCS Ca' Granda Ospedale Maggiore Policlinico, \\ Milano, Italy
}

Correspondence should be addressed to: Kinga Diana Siczek; kdiana77@libero.it

Received date: 10 January 2014; Accepted date: 3 July 2014; Published date: 31 December 2014

Academic Editor: Ion Codreanu

Copyright (c) 2014. Kinga Diana Siczek, Paola Faggioli, Emanuela Venegoni, Luigia Florimonte, Marco Carletto, Fabio Cammelli, Cristina Songini, Alberto Vignati, Rossella Stoico, Antonino Mazzone, Lorenzo S. Maffioli. Distributed under Creative Commons CC-BY 3.0

\begin{abstract}
Objective: Evaluate the diagnostic accuracy of PET in patients with fever of unknown origin (FUO) and in patients with suspected and/or proved large vessel vasculitis (LVV). Patients and methods: 72 patients (49 females, 23 males; mean age 63 y, range 23-82) were prospectively examined with PET between February 2010 and April 2013; 42 with suspected LVV, 15 with LVV during steroid therapy, and 15 with fever of unknown origin (FUO). Follow-up scans were performed in 19 patients. A semi-quantitative analysis [Vessel SUVmax]/[liver SUVmax] was performed to rate the disease in a 4-step system. PET scans were compared with temporal artery biopsy and MRI and/or angio-CT of a specific region. Results: Pathological ${ }^{18 F-F D G ~(F D G) ~ u p t a k e ~ w a s ~ o b s e r v e d ~ i n ~ 30 / 72 ~(42 \%) ~ p a t i e n t s, ~ a n d ~}$ negative in $42 / 72$ (58\%) patients. In 42 patients with suspected LVV 9 (21\%) were positive, and $33(79 \%)$ negative; 11 (73\%) of the 15 steroid-treated patients with LVV were positive, and $4(27 \%)$ negative; $10(67 \%)$ of the 15 patients with FUO were positive and $5(33 \%)$ negative. PET had an overall sensitivity of 87\% [95\% confidence interval (CI) 79-95\%], a specificity of $95 \%$ (95\% CI 90-100\%), a positive predictive value of $93 \%$ (95\% CI 87-99\%) and a negative predictive value of $91 \%$ (95\% CI 84-97\%). The diagnostic accuracy of PET was $92 \%$ (95\% CI 85-98\%). Conclusions: ${ }^{18}$ F-FDG PET/CT especially that based on semi quantitative analysis is a sensitive and specific imaging tool for the diagnosis of LVV but also for therapy monitoring and follow up.
\end{abstract}

Keywords: vasculitis, FDG-PET/CT, corticosteroid therapy

Cite this Article as: Kinga Diana Siczek, Paola Faggioli, Emanuela Venegoni, Luigia Florimonte, Marco Carletto, Fabio Cammelli, Cristina Songini, Alberto Vignati, Rossella Stoico, Antonino Mazzone and Lorenzo S. Maffioli (2014)," Role of 18F-FDG PET-CT in vasculitis: a prospective study ", JMED Research, Vol. 2014 (2014), Article ID 389734, DOI: 10.5171/2014.389734 


\section{Introduction}

The diagnosis of large vessel vasculitis (LVV), as Giant cell arteritis (GCA) and Takayasu's arteritis (TA), in clinical practice is often a difficult task, and it usually requires time and financial resources in order to define the characteristics of disease in patients with nonspecific symptoms and elevated inflammatory markers. In particular, two subsets of patients are a diagnostic challenge: 1) patients with fevers of unknown origin (FUOs), who can be classified into 4 main clinical categories: infectious, malignant, rheumaticinflammatory and miscellaneous disorders (Meller et al. 2007, and Roth et al. 2003); 2) patients with increased inflammatory markers and systemic signs/symptoms in the absence of infections or tumors, (Gaeta et al. 2006).

Revised International Chapel Hill Consensus Conference Nomenclature of Vasculitis (2013), (Jennette et al. 2012), classified vasculitis by:

1) Vessel diameter

2) Presence of granulomatous lesions

3) Presence of antineutrophil cytoplasmic antibodies (ANCA).

Standard diagnostic procedures include laboratory tests, biopsy, angiography, ultrasound and magnetic resonance angiography (Fuchs 2012), although these conventional diagnostic methods are not adequate to make a definitive diagnosis in approximately $50 \%$ of patients in this small group.

In studies by Mukhtyar (2009) and Basu (2012), laboratory tests, including erythrocyte sedimentation rate (ESR), Creactive protein (PCR) and ANCA antibodies, were found to be nonspecific for large vessel vasculitis diagnosis.

Conventional imaging techniques such as Computed Tomography - (CT), angioComputed Tomography (angio-CT), Magnetic Resonance (MR) and/or angio Magnetic Resonance (angio-MR), ultrasound, and biopsy can be invasive, operator dependent, and often biased during disease duration and therapy. In particular MR takes relatively long time, can study only one region at time and cannot be performed in patients with pace maker. Angio-CT cannot be performed in patients with kidney disease because of the contrast.

Positron emission tomography-computed tomography (PET/CT), instead, is a noninvasive, operator independent, metabolic imaging modality based on the regional distribution of the glucose analogue (fluorine-18 fluorodeoxyglucose 18F-FDG) (Fuchs 2012) and can be performed in all kinds of patients. In retrospective studies by Cao (2012), Qiu (2012), Watts (2009), Tezuka (2012), Talarico (2012) and Pipitone (2008) the performance of FDG PET/CT to diagnose vasculitis, rheumatic diseases, inflammatory diseases, and peritoneal fibrosis was evaluated usually enrolling a low amount of patients.

Sheng (2011), Cao (2012) and Qiu (2012) suggested that for the correct diagnosis with PET the addition of conventional imaging techniques may generally offer improved diagnostic accuracy compared with current standards of practice.

The aim of this study was the diagnostic accuracy of PET in patients with fever of unknown origin (FUO) and in patients with suspected and/or proved large vessel vasculitis treated with and without immunosuppressive drugs, and the possibility of the use of PET in these diseases' monitoring and treatment.

\section{Patients and Methods}

\section{Patients}

72 patients (49 females, 23 males; mean age $63 \mathrm{y}$, range 23-82), who were referred to our interdisciplinary centre in the Department of Rheumatology, were prospectively examined with PET imaging between February 2010 and April 2013; 42 with suspected large vessel vasculitis (LVV) 15 with (LVV) during steroid therapy (over $5 \mathrm{mg}$ of prednisone/day) and 15 with fever 
of unknown origin (FUO). Follow-up scans were performed in 19 patients. Diagnoses were based on the American College of Rheumatology (ACR) classification criteria, laboratory tests, conventional imaging (angio-MRI and/or angio-CT, and/or ultrasound), on temporal artery biopsy in some patients and the exclusion of other diagnoses (Tab 1).

According to validated diagnostic criteria for vasculitis and for FUO, these are the main criteria we used to have our patients undergo PET imaging $(13,4)$, (Tab. 2 and Tab. 3).

Table 1: Patient studied with PET/CT

\begin{tabular}{|c|c|c|c|c|c|c|c|c|c|}
\hline $\mathbf{P}$ & Age & Sex & Pathology & Laboratory & $\begin{array}{c}\text { Cortisonic } \\
\text { therapy }\end{array}$ & $\begin{array}{c}\text { PET/ } \\
\text { CT }\end{array}$ & $\begin{array}{c}\text { FU } \\
\text { PET/CT }\end{array}$ & $\begin{array}{l}\text { Bio } \\
\text { psy }\end{array}$ & C.I. \\
\hline 1 & 63 & $\bar{F}$ & Vasculitis in tp & $\overline{E S R} \uparrow \mathrm{CRP} \uparrow$ & Yes $25 \mathrm{mg}$ & Pos & Neg & Yes & Yes \\
\hline 2 & 60 & $M$ & Aortitis in tp & - & Yes $25 \mathrm{mg}$ & Pos & $\mathrm{Neg}$ & Yes & Yes \\
\hline 3 & 42 & F & $\begin{array}{l}\text { Suspected } \\
\text { aortitis }\end{array}$ & $\overline{\mathrm{ESR} \uparrow \mathrm{CRP} \uparrow}$ & No & Neg & - & No & Yes \\
\hline 4 & 63 & $\bar{F}$ & $\begin{array}{l}\text { Suspected } \\
\text { arteritis in AR }\end{array}$ & ESR $\uparrow C R P \uparrow$ & No & Pos & - & No & Yes \\
\hline 5 & 27 & $\mathrm{~F}$ & $\begin{array}{l}\text { Suspected } \\
\text { aortitis }\end{array}$ & - & No & Neg & Pos/neg & No & Yes \\
\hline 6 & 73 & $M$ & $\begin{array}{l}\text { Suspected } \\
\text { arteritis in PMR }\end{array}$ & - & No/7.5 mg & Neg & Neg/neg & No & Yes \\
\hline 7 & 78 & $\bar{F}$ & $\begin{array}{l}\text { Suspected } \\
\text { arteritis }\end{array}$ & ESR $\uparrow C R P \uparrow$ & No & Pos & - & No & Yes \\
\hline 8 & 59 & $\mathrm{M}$ & FUO & ESR $\uparrow C R P \uparrow$ & $\begin{array}{l}\text { No/yes/yes/ } \\
\text { yes }\end{array}$ & Pos & $\begin{array}{l}\text { Neg/neg } \\
\text { /neg }\end{array}$ & No & Yes \\
\hline 9 & 66 & $\bar{F}$ & Vasculitis in tp & 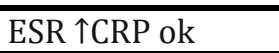 & Yes/yes & Pos & Pos & No & Yes \\
\hline 10 & 79 & $\mathrm{~F}$ & Vasculitis in tp & $\overline{E S R \uparrow C R P \uparrow}$ & Yes $2,5 \mathrm{mg}$ & Pos & - & Yes & Yes \\
\hline$\overline{11}$ & 44 & $M$ & $\begin{array}{l}\text { Suspected } \\
\text { vasculitis }\end{array}$ & - & No & Neg & - & No & - \\
\hline 12 & 56 & $F$ & $\begin{array}{l}\text { Suspected } \\
\text { vasculitis }\end{array}$ & - & No & $\mathrm{Neg}$ & - & No & Yes \\
\hline 13 & 77 & $\bar{F}$ & FUO & - & No & Neg & - & No & Yes \\
\hline 14 & 80 & $\bar{F}$ & $\begin{array}{l}\text { Suspected } \\
\text { vasculitis }\end{array}$ & ESR $\uparrow C R P \uparrow$ & No/yes $10 \mathrm{mg}$ & Pos & Neg & No & Yes \\
\hline 15 & 59 & $M$ & $\begin{array}{l}\text { Suspected } \\
\text { vasculitis }\end{array}$ & ESR $\uparrow \mathrm{CRP} \uparrow$ & No & $\mathrm{Neg}$ & - & No & Yes \\
\hline$\overline{16}$ & 23 & $\bar{F}$ & $\begin{array}{l}\text { Suspected } \\
\text { vasculitis }\end{array}$ & $\overline{E S R} \uparrow C R P \uparrow$ & No & Neg & - & No & $\overline{\text { Yes }}$ \\
\hline 17 & 48 & $\mathrm{~F}$ & FUO & - & No & Neg & - & No & Yes \\
\hline 18 & 79 & $\mathrm{M}$ & Horton a in tp & - & - & $\mathrm{Neg}$ & - & No & Yes \\
\hline 19 & 77 & $\mathrm{~F}$ & $\begin{array}{l}\text { Suspected } \\
\text { vasculitis }\end{array}$ & - & No & $\mathrm{Neg}$ & - & - & - \\
\hline 20 & 77 & $\bar{F}$ & FUO in PMR & $\overline{\mathrm{ESR} \uparrow \mathrm{CRP} \uparrow}$ & No & $\mathrm{Neg}$ & - & Yes & Yes \\
\hline 21 & 69 & $\bar{F}$ & $\begin{array}{l}\text { Suspected } \\
\text { vasculitis }\end{array}$ & - & No & $\mathrm{Neg}$ & - & No & $\overline{\text { Yes }}$ \\
\hline 22 & 61 & $F$ & FUO & $\mathrm{ESR} \uparrow \mathrm{CRP} \uparrow$ & No/yes & Pos & $\mathrm{Neg}$ & No & Yes \\
\hline 23 & 65 & $\bar{F}$ & $\begin{array}{l}\text { Suspected } \\
\text { arteritis }\end{array}$ & - & No & Neg & - & No & $\overline{\text { Yes }}$ \\
\hline
\end{tabular}




\begin{tabular}{|c|c|c|c|c|c|c|c|c|c|}
\hline $\mathbf{P}$ & Age & $\begin{array}{l}\text { Se } \\
\mathrm{x}\end{array}$ & Pathology & Laboratory & $\begin{array}{c}\text { Cortisonic } \\
\text { therapy }\end{array}$ & $\begin{array}{c}\text { PET/ } \\
\text { CT }\end{array}$ & $\begin{array}{c}\text { FU } \\
\text { PET/CT }\end{array}$ & $\begin{array}{l}\text { Bio } \\
\text { psy }\end{array}$ & C.I. \\
\hline 24 & 77 & $\mathrm{M}$ & Arteritis in tp & $\mathrm{ESR} \uparrow \mathrm{CRP} \uparrow$ & $\begin{array}{ll}\text { Yes } & 15 \\
\mathrm{mg} / \mathrm{No} & \\
\end{array}$ & Pos & Neg & No & Yes \\
\hline 25 & 62 & $\mathrm{~F}$ & FUO & ESR $\uparrow C R P \uparrow$ & No/yes/yes & Pos & Pos/pos & No & Yes \\
\hline 26 & 72 & $\mathrm{~F}$ & Suspected arteritis & - & No & Neg & - & No & Yes \\
\hline 27 & 73 & $\bar{M}$ & Suspected vasculitis & $\begin{array}{l}\text { ANA pos, ANCA, } \\
\text { antiDNA,ENA neg }\end{array}$ & No & Neg & - & No & Yes \\
\hline 28 & 60 & $\mathrm{~F}$ & Horton a in tp & - & Yes/yes $5 \mathrm{mg}$ & Pos & $\mathrm{Neg}$ & No & Yes \\
\hline 29 & 50 & $\mathrm{~F}$ & Suspected vasculitis & $\overline{E S R \uparrow C R P \uparrow}$ & No & Neg & - & No & Yes \\
\hline 30 & 68 & $\bar{M}$ & Suspected vasculitis & ESR $\uparrow C R P \uparrow$ & No & $\mathrm{Neg}$ & - & Yes & Yes \\
\hline 31 & 64 & $\bar{F}$ & FUO & $\overline{E S R} \uparrow \mathrm{CRP} \uparrow$ & $\begin{array}{l}\text { No/yes } 20 \\
\text { mg/yes } 5 \text { mg }\end{array}$ & Pos & Neg/neg & No & Yes \\
\hline 32 & 55 & $\mathrm{M}$ & FUO & ESR $\uparrow C R P \uparrow$ & $\begin{array}{ll}\text { No/yes } & 7.5 \\
\mathrm{mg} & \\
\end{array}$ & Pos & Neg & No & Yes \\
\hline 33 & 45 & $\mathrm{M}$ & Suspected vasculitis & - & No & Neg & - & No & - \\
\hline 34 & 38 & $\mathrm{~F}$ & Suspected vasculitis & - & No & Neg & - & No & - \\
\hline 35 & 72 & $\bar{M}$ & Vasculitis in tp & - & Yes 7,5 mg & $\mathrm{Neg}$ & - & No & Yes \\
\hline 36 & 58 & $\mathrm{~F}$ & Suspected vasculitis & ESR $\uparrow C R P \uparrow$ & No & $\mathrm{Neg}$ & - & No & Yes \\
\hline 37 & 63 & $\mathrm{~F}$ & FUO & $\mathrm{ESR} \uparrow \mathrm{CRP} \uparrow$ & No & $\mathrm{Neg}$ & - & No & Yes \\
\hline 38 & 57 & $\mathrm{~F}$ & Suspected vasculitis & ESR $\uparrow C R P \uparrow$ & No & $\mathrm{Neg}$ & - & No & Yes \\
\hline 39 & 82 & $\mathrm{~F}$ & FUO & ANCA pos & $\begin{array}{lr}\text { Yes } & 25 \\
\mathrm{mg} / \text { yes } & 5 \\
\mathrm{mg} / \text { yes } & 7.5 \\
\mathrm{mg} & \\
\end{array}$ & Pos & Neg/neg & No & Yes \\
\hline 40 & 76 & $\mathrm{M}$ & FUO & - & No/no & Pos & $\mathrm{Neg}$ & No & Yes \\
\hline 41 & 70 & $\mathrm{M}$ & $\begin{array}{l}\text { Suspected vasculitis } \\
\text { in AP }\end{array}$ & $\mathrm{ESR} \uparrow \mathrm{CRP} \uparrow$ & Yes $5 \mathrm{mg}$ & Neg & - & No & Yes \\
\hline 42 & 30 & $\mathrm{~F}$ & FUO & $\mathrm{ESR} \uparrow \mathrm{CRP} \uparrow$ & No & $\mathrm{Neg}$ & - & No & Yes \\
\hline 43 & 38 & $\mathrm{~F}$ & Suspected vasculitis & - & - & $\mathrm{Neg}$ & - & No & Yes \\
\hline 44 & 72 & $\mathrm{~F}$ & $\begin{array}{l}\text { Suspected vasculitis } \\
\text { in PMR }\end{array}$ & - & - & Neg & - & No & Yes \\
\hline 45 & 68 & $\mathrm{M}$ & Vasculitis in tp & ANCA pos & Yes $5 \mathrm{mg}$ & $\mathrm{Neg}$ & - & No & Yes \\
\hline 46 & 24 & $\mathrm{M}$ & Suspected vasculitis & - & No & $\mathrm{Neg}$ & - & No & Yes \\
\hline 47 & 49 & $\mathrm{~F}$ & Suspected vasculitis & $\begin{array}{l}\text { CRP ok ANA, ENA, } \\
\text { ANCA neg, }\end{array}$ & Yes & Neg & - & No & Yes \\
\hline 48 & 73 & $\mathrm{~F}$ & $\begin{array}{l}\text { Suspected vasculitis } \\
\text { in AR }\end{array}$ & $\begin{array}{l}\text { ANA, ENA, anti DNA } \\
\text { neg; }\end{array}$ & No & Neg & - & No & Yes \\
\hline 49 & 81 & $\mathrm{M}$ & Suspected aortitis & $\mathrm{ESR} \uparrow \mathrm{CRP} \uparrow$ & No & Pos & - & No & Yes \\
\hline 50 & 69 & $\mathrm{~F}$ & Suspected vasculitis & ESR $\uparrow C R P \uparrow$ & Yes $4 \mathrm{mg}$ & Neg & - & No & Yes \\
\hline 51 & 72 & $\mathrm{~F}$ & Suspected vasculitis & ESR $\uparrow C R P \uparrow A N C A$ pos & Yes $4 \mathrm{mg}$ & Neg & - & No & Yes \\
\hline 52 & 72 & $\mathrm{~F}$ & Suspected vasculitis & ESR $\uparrow C R P \uparrow$ & No & Neg & - & No & Yes \\
\hline 53 & 75 & $\bar{M}$ & Suspected vasculitis & $\overline{E S R \uparrow C R P \uparrow}$ & Yes $5 \mathrm{mg}$ & Pos & - & No & Yes \\
\hline 54 & 60 & $\mathrm{~F}$ & Suspected vasculitis & ESR $\uparrow C R P \uparrow$ & No & $\mathrm{Neg}$ & - & No & Yes \\
\hline 55 & 72 & $\mathrm{M}$ & Suspected vasculitis & ANA pos & Yes $5 \mathrm{mg}$ & Neg & - & No & Yes \\
\hline
\end{tabular}




\begin{tabular}{|c|c|c|c|c|c|c|c|c|c|}
\hline $\mathbf{P}$ & Age & Se & Pathology & Laboratory & $\begin{array}{l}\text { Cortisonic } \\
\text { therapy }\end{array}$ & $\begin{array}{l}\text { PET/ } \\
\text { CT }\end{array}$ & $\begin{array}{c}\text { FU } \\
\text { PET/CT }\end{array}$ & $\begin{array}{l}\text { Bio } \\
\text { psy }\end{array}$ & C.I. \\
\hline 56 & 79 & $\mathrm{~F}$ & Suspected vasculitis & $\begin{array}{l}\text { ENA pos; ANA, anti } \\
\text { DNA neg }\end{array}$ & No & Pos & - & No & Yes \\
\hline 57 & 67 & $\mathrm{~F}$ & Suspected vasculitis & $\begin{array}{l}\text { ESR ok ANA pos, ENA, } \\
\text { antiDNA neg }\end{array}$ & No & Neg & - & No & Yes \\
\hline 58 & 70 & $\mathrm{M}$ & Suspected vasculitis & $\mathrm{ESR} \uparrow$ & No & Neg & - & - & Yes \\
\hline 59 & 64 & $\bar{F}$ & Suspected vasculitis & $\begin{array}{l}\text { ESR } \uparrow C R P \uparrow ~ A N A, E N A, \\
\text { anti DNA neg }\end{array}$ & No & Neg & - & No & Yes \\
\hline 60 & 57 & $\mathrm{~F}$ & Vasculitis in tp & ESR $\uparrow \mathrm{CRP} \uparrow$ & Yes & Neg & - & No & Yes \\
\hline 61 & 74 & $\bar{F}$ & FUO & ESR $\uparrow$ & No & Pos & - & $\mathrm{Nd}$ & $\overline{\text { Yes }}$ \\
\hline 62 & 63 & $\bar{F}$ & $\overline{\text { FUO }}$ & ESR $\uparrow$, ANA pos & $\begin{array}{ll}\text { No/yes } & 25 \\
\mathrm{mg} / \text { yes } & 25 \\
\mathrm{mg} & \\
\end{array}$ & Pos & Pos/pos & No & Yes \\
\hline 63 & 62 & $\mathrm{~F}$ & Suspected vasculitis & - & No & Pos & - & - & Yes \\
\hline 64 & 65 & $\bar{F}$ & Suspected vasculitis & - & No & Pos & - & - & Yes \\
\hline 65 & 70 & $\bar{F}$ & Vasculitis in tp & - & Yes & Pos & - & - & $\overline{\text { Yes }}$ \\
\hline 66 & 58 & $\bar{F}$ & Vasculitis in tp & - & Yes & Pos & - & - & $\overline{\text { Yes }}$ \\
\hline 67 & 63 & $\bar{M}$ & Vasculitis in tp & - & Yes & Pos & - & - & Yes \\
\hline 68 & 63 & $\bar{M}$ & Vasculitis in tp & - & Yes & Pos & - & - & Yes \\
\hline 69 & 77 & $\bar{F}$ & Suspected vasculitis & $\overline{E S R ~ \uparrow C R P ~ o k ~}$ & Yes/no & Pos & $\mathrm{Neg}$ & No & - \\
\hline 70 & 68 & $\bar{M}$ & Vasculitis in tp & $\begin{array}{l}\text { ESR } \uparrow \text { CRP ok, ANA, } \\
\text { ENA, ANCA, antiDNA } \\
\text { neg }\end{array}$ & Yes $25 \mathrm{mg}$ & Pos & - & No & Yes \\
\hline 71 & 70 & $\mathrm{~F}$ & FUO & $\begin{array}{l}\text { ESR } \uparrow \text { CRP } \uparrow \text { ANA pos, } \\
\text { ENA neg }\end{array}$ & $\begin{array}{lr}\text { Yes } & 25 \\
\mathrm{mg} / \text { yes } & 7.5 \\
\mathrm{mg} & \\
\end{array}$ & Pos & Pos & No & Yes \\
\hline 72 & 67 & $\bar{F}$ & Suspected vasculitis & 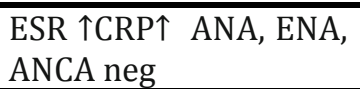 & $\begin{array}{l}\text { No/yes/yes } 5 \\
\text { mg }\end{array}$ & Neg & Neg/neg & No & Yes \\
\hline
\end{tabular}

$\mathrm{P}=$ patients; FU = follow up with PET/CT; C. I. = conventional imaging (angio-CT, angio-RM, ultrasound); F = female; $\mathrm{M}$ = male; $t \mathrm{p}=$ therapy (corticosteroid therapy); Pos = positive; Neg = negative; ESR = Erythrocyte sedimentation rate; $\mathrm{CRP}=\mathrm{C}$-Reactive protein; ANA = antinuclear antibodies; antiDNA = native DNA antibodies; ENA = Extractable nuclear antigens; $\mathrm{ANCA}=$ Antineutrophil cytoplasmic antibodies; FUO = Fever of unknown origin

Table 2: Vasculitis diagnostic main criteria

\begin{tabular}{|l|l|}
\hline 1) Systemic & weight loss, nausea, fatigue, fever, malaise, night sweats \\
\hline 2) Temporal arteritis & $\begin{array}{l}\text { jaw claudication, temporal artery thickening or local headache, } \\
\text { eyesight abnormalities, myalgia and polymyalgia-like rheumatic } \\
\text { symptoms }\end{array}$ \\
\hline 3) Cardiovascular & $\begin{array}{l}\text { arterial claudication, peripheral pulse weakness, echocardiographic } \\
\text { ultrasound abnormalities }\end{array}$ \\
\hline 4) Pulmonary & non infectious pulmonary infiltrates and interstitial lung diseases \\
\hline 5) Neurological & dizziness, headache, ischemic attack, polyneuropathy \\
\hline 6) Laboratory tests & $\begin{array}{l}\text { increased C-Reactive Protein (CRP), increased Erythrocyte } \\
\text { Sedimentation Rate (ESR), increased creatinine, positivity to } \\
\text { Antinuclear Antibodies (ANA), native-DNA Antibodies (anti-n-DNA } \\
\text { Ab), Extractable Nuclear Antigens (ENA), Antineutrophil Cytoplasmic } \\
\text { Antibodies (ANCA) }\end{array}$ \\
\hline
\end{tabular}

Kinga Diana Siczek, Paola Faggioli, Emanuela Venegoni, Luigia Florimonte, Marco Carletto, Fabio Cammelli, Cristina Songini, Alberto Vignati, Rossella Stoico, Antonino Mazzone and Lorenzo S. Maffioli (2014), JMED Research, DOI: 10.5171/2014.389734 
Table 3: FUO main diagnostic criteria (PET should be performed before bone marrow biopsy).

1) Negative bacterial cultures

2) Negative serologic tests

3) Negative total body CT scans

4) Negative echocardiography

The study's endpoint was the diagnostic accuracy of PET for large vessel vasculitis (LVV) patients treated with and without immunosuppressive drugs, and the possibility of the use of PET for disease monitoring and treatment.

All scans were acquired after a 6hour fast, using an integrated PET/CT camera (PHILIPS GEMINI TF), equipped with a fullring dedicated PET scanner and a sixteen slice CT scanner. The serum glucose level was measured before ${ }^{18} \mathrm{~F}-\mathrm{FDG}$ administration in all patients and was below 120 mg/dl (Glucometer Breeze 2, Bayer Diagnostics). PET scans were performed $60 \mathrm{~min}$ after intravenous injection using a venous line of 3,7 MBq/Kg body weight of ${ }^{18} \mathrm{~F}$-FDG. Low dose CT (max $120 \mathrm{Kv}, 100 \mathrm{mAs}$ ) and PET scans were performed from the base of the skull to the mid thigh. We performed whole-body scans using 3D acquisition with 7-8 contiguous bed positions ( $2 \mathrm{~min} / \mathrm{bed})$. Iterative ordered subset expectation maximization (OSEM) reconstruction algorithm was used to obtain $512 \times 512$ format trans-axial slices of 4-5 mm thickness; CT attenuation correction was performed. Philips Imaging software was used to view and analyze the reconstructed images in PET-CT fusion modality.

\section{PET Imaging and Statistical analysis}

All scans were assessed by a panel of board certified nuclear medicine specialists.

PET scans were analysed visually in a descriptive manner, as well as by a quantitative computed method.

First visual analysis was performed. Thereafter, image readings were divided into three groups: normal vessel uptake, abnormal uptake in vessels with atherosclerotic plaque, and abnormal uptake in vessel without atherosclerotic plaque (Fig 1a, 1b, 1c).

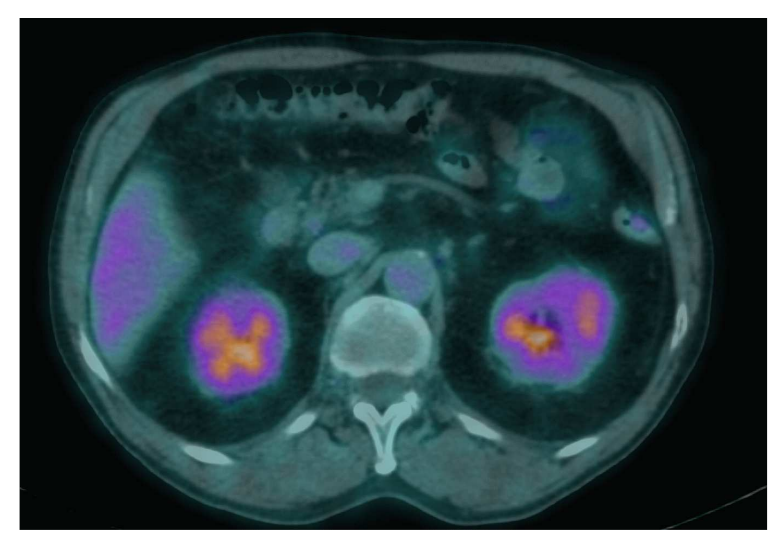

Figure 1A: Regular aorta uptake

Kinga Diana Siczek, Paola Faggioli, Emanuela Venegoni, Luigia Florimonte, Marco Carletto, Fabio Cammelli, Cristina Songini, Alberto Vignati, Rossella Stoico, Antonino Mazzone and Lorenzo S. Maffioli (2014), JMED Research, DOI: 10.5171/2014.389734 


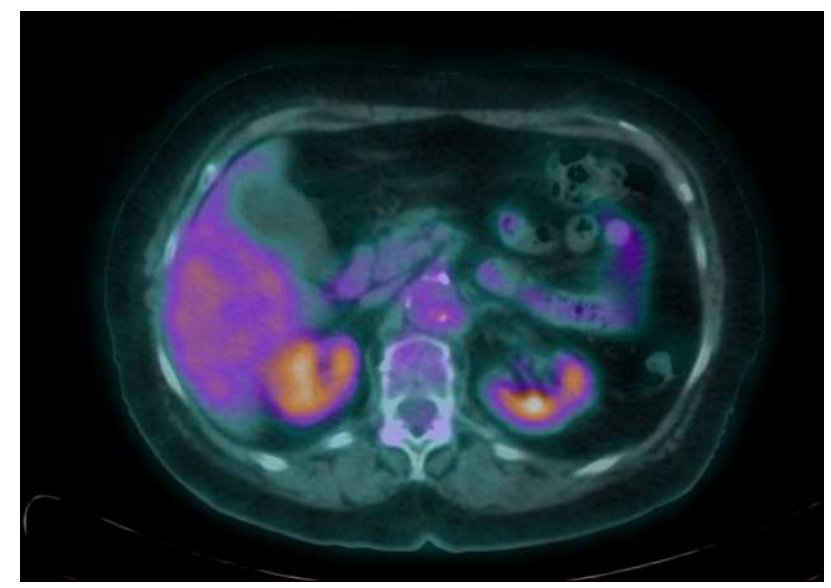

Figure 1B: Atherosclerotic plaque in aorta irregular uptake Vessel/liver ratio 1

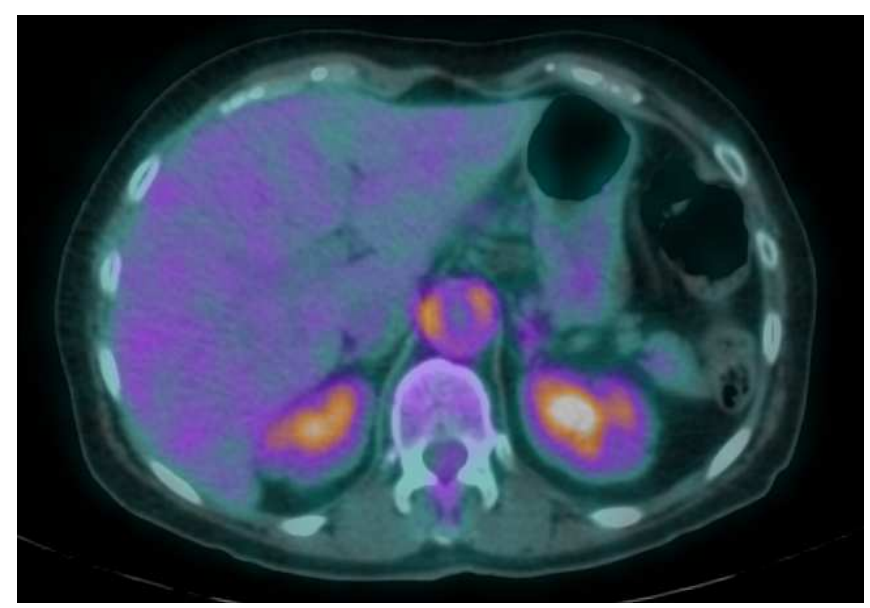

Figure 1C: Vasculitis vessel/liver ratio 1,8

Figure 1: FDG uptake in normal aorta wall (FIG 1A); FDG uptake in atherosclerotic wall of aorta (Fig 1 B): calcific plaque were considered as false positives; FDG uptake in vasculitis (Fig 1 C).

For quantitative evaluation, region of interest (ROI) was placed over nine defined vascular areas (ascending thoracic aorta, descending thoracic aorta, abdominal aorta, right and left subclavian arteries, right and left external carotid arteries, right and left common iliac arteries) and right hepatic lobe.

Using regions of interest SUVmax vessel to liver ratio was calculated and rated into 4 Grades. The means and standard deviations were calculated for orderly vessel/liver uptake ratio distributions, divided into two groups: negative $(<1.0)$ and highly positive $(\geq 1.0)$. For each group, the normal distribution was computed. Statistical analysis calculated different intervals (vessel/liver ratio < 0.9 was considered grade 0 ; vessel/liver ratio between 0.9 and 1 was considered grade I; vessel/liver ratio between 1 and1.1 was considered grade II; vessel/liver ratio $\geq 1.1$ was considered grade III). Grade II and III were considered pathological. 
Sensitivity, specificity, positive predictive value, negative predictive value and diagnostic accuracy of FDG PET/CT were calculated using clinical criteria and conventional imaging as the reference standard.

\section{Results}

The reference point for highly positive cases ( $\geq 1.1$, blue area) was identified by the overlap of the two normal distributions. The mildly and moderately positive grades (mild: range $0.9-<1.0$, orange area, and moderate: range $1.0-<1.1$, green area) were defined by the presence of the positive distribution tail subtending the negative normal distribution (Fig2).

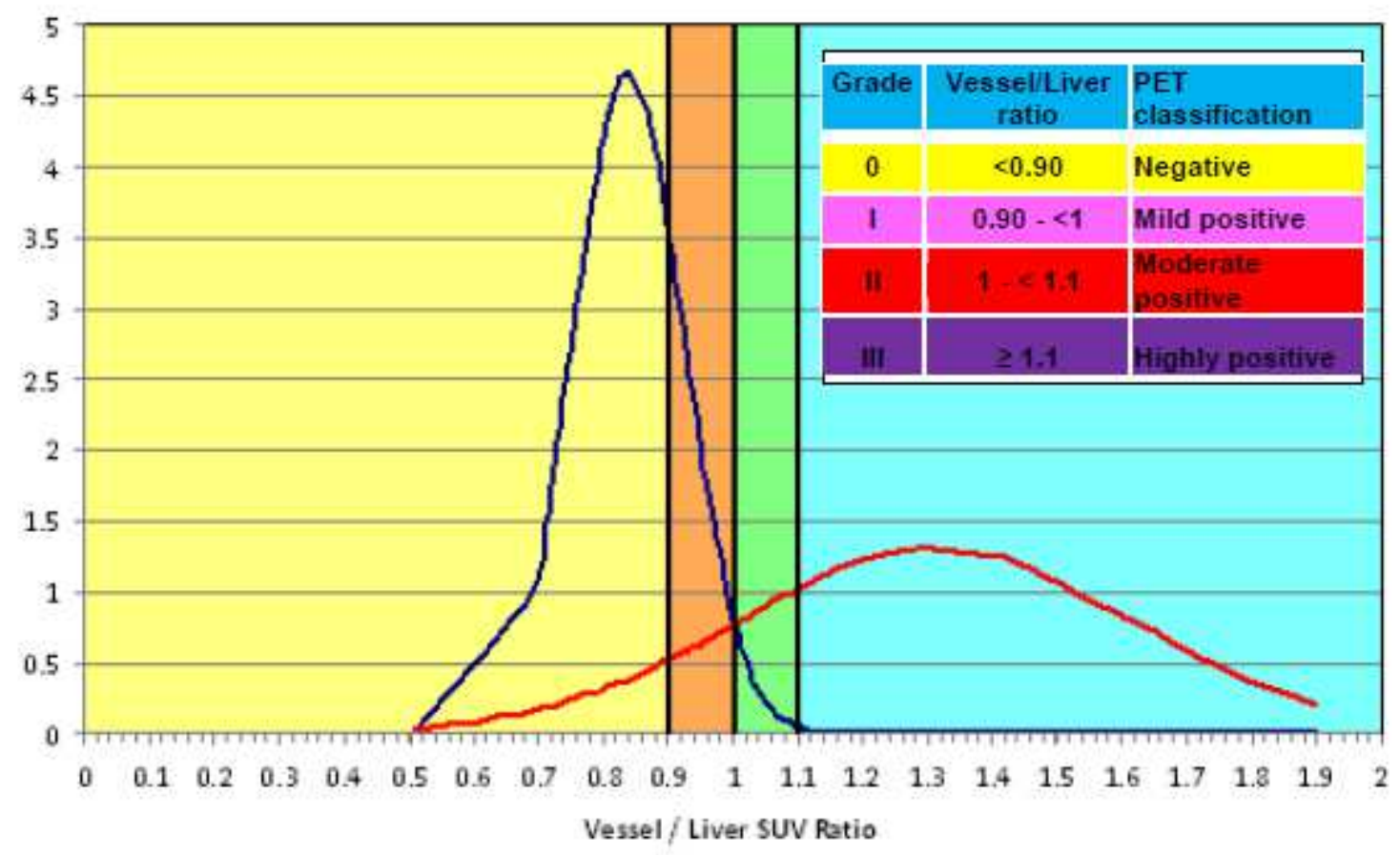

Figure 2: Normal distribution of SUVmax vessel/liver ratio; a semiquantitative analysis on (vessel SUV max)/(liver SUV max) was performed to rate the disease into a 4-step system

Using a semiquantitative analysis based on SUV max vessel to liver ratio, PET scans were considered positive for large vessel vasculitis in $30 / 72(42 \%)$ patients, and negative in $42 / 72(58 \%)$ patients. In 42 on PET scan, and 4 (27\%) negative; in 15 patients with fever of unknown origin patients with suspected LVV: 9 (21\%) were positive on PET scan, and 33 (79\%) were negative; in 15 patients with LVV during steroid therapy: 11 (73\%) were positive

(FUO): 10 (67\%) were PET positive and 5 (33\%) negative (Table 4, Fig 3). 
Table 4: FDG PET/CT results

\begin{tabular}{|c|c|c|c|}
\hline FDG PET & Suspected vasculitis & Vasculitis in steroid therapy & FUO \\
\hline Positive (Grade II - III) & 9 & 11 & 10 \\
\hline Negative (Grade 0 - I) & 33 & 4 & 5 \\
\hline Total & 42 & 15 & 15 \\
\hline
\end{tabular}
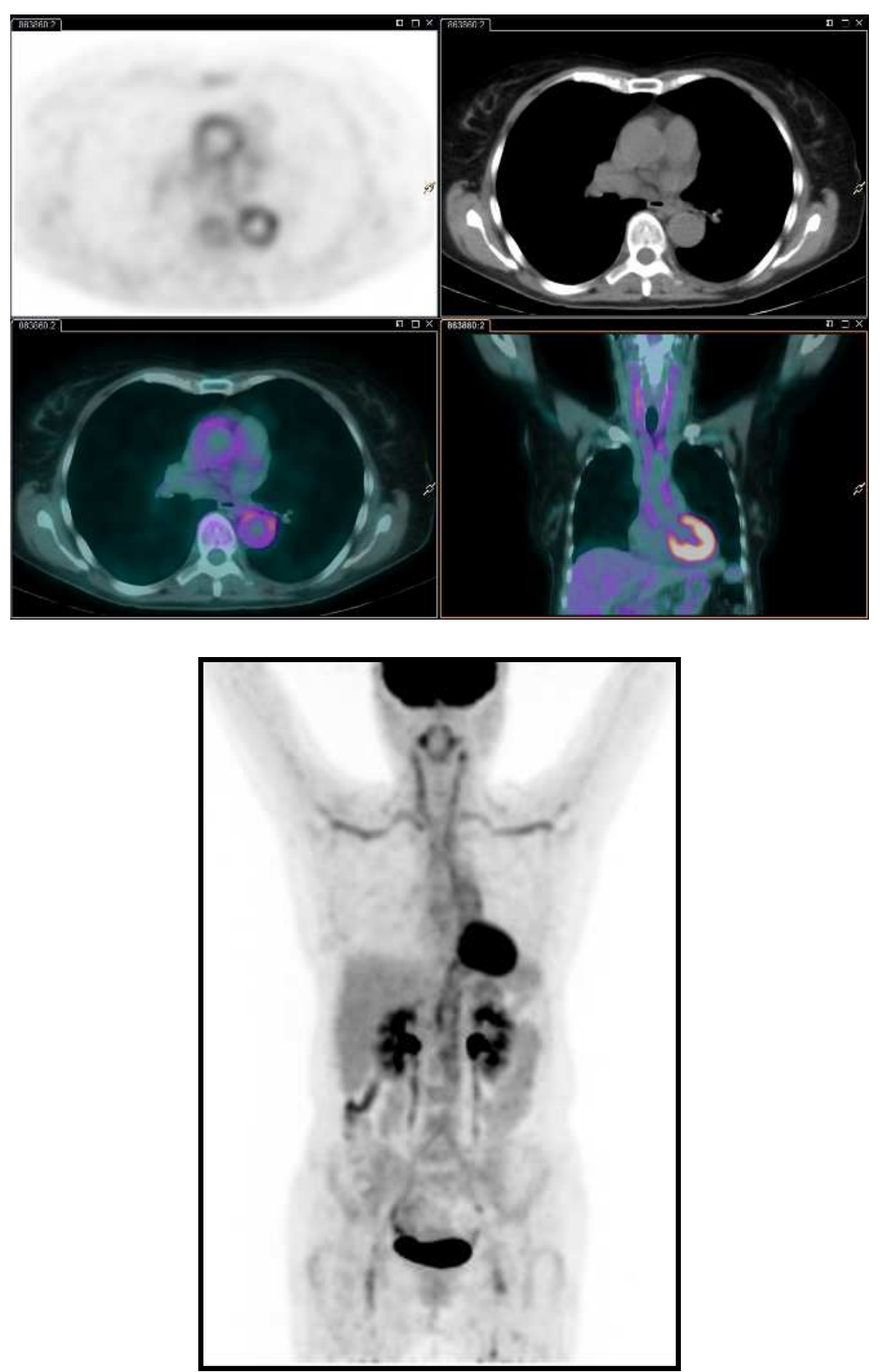

Kinga Diana Siczek, Paola Faggioli, Emanuela Venegoni, Luigia Florimonte, Marco Carletto, Fabio Cammelli, Cristina Songini, Alberto Vignati, Rossella Stoico, Antonino Mazzone and Lorenzo S. Maffioli (2014), JMED Research, DOI: 10.5171/2014.389734 

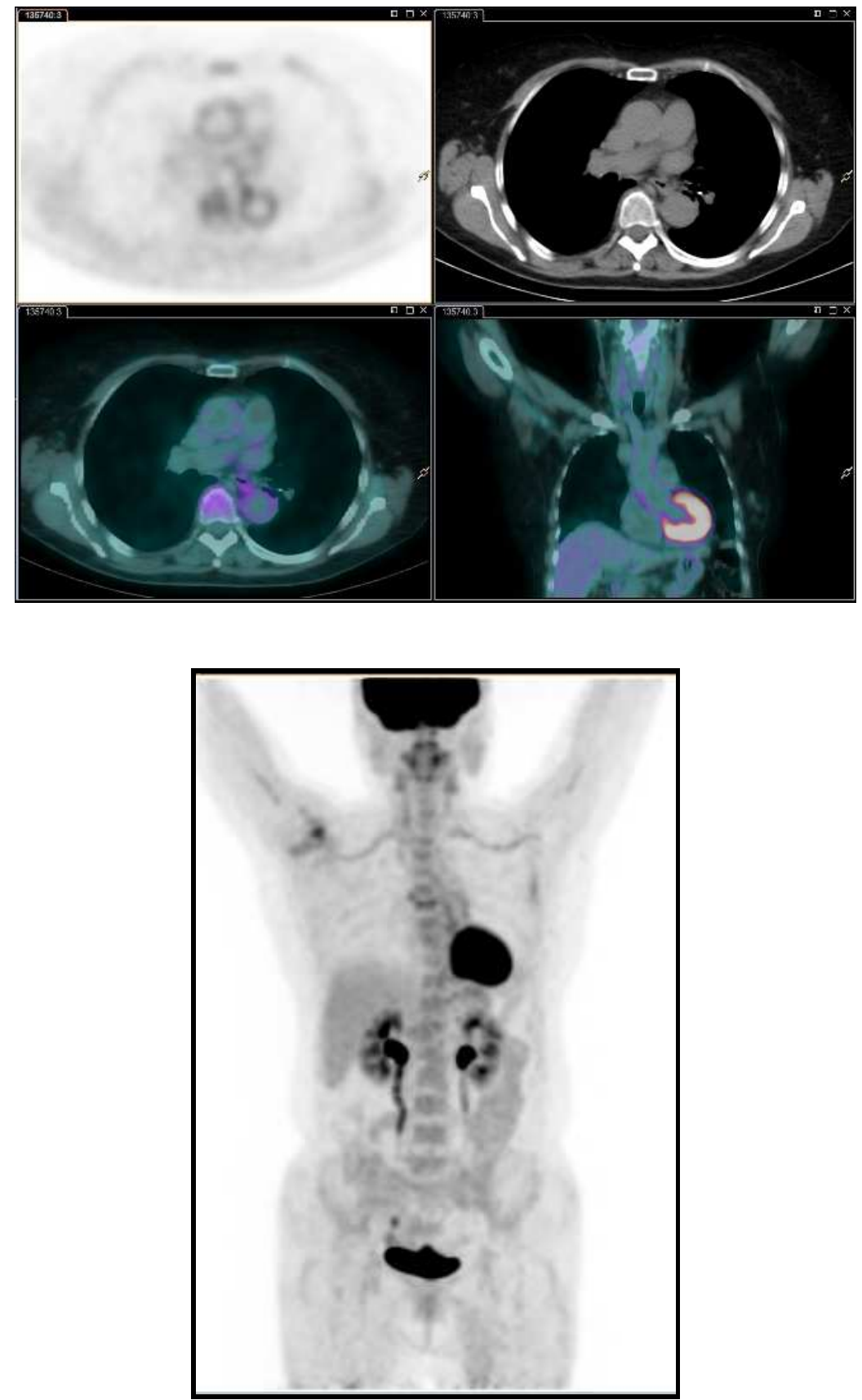

Figure 3: FUO pre-therapy: vessel to liver ratio 1,6 (Fig 3A1-2); FUO post-therapy: vessel to liver ratio 1 (Fig 3B1-2).

Considering clinical criteria and conventional imaging, PET had an overall sensitivity of $87 \% \quad[95 \%$ confidence interval (CI) 79-95\%], a specificity of $95 \%$ (95\% CI 90-100\%), a positive predictive value of $93 \%$ (95\% CI $87-99 \%)$ and a negative predictive value of $91 \%$ (95\% CI $84-97 \%)$. The diagnostic accuracy of PET was $92 \%$ (95\% CI 85-98\%).
As reported by Fuchs et al.(2012), specificity and sensitivity of PET can be lower $(64.5 \%)$ in patients on immunosuppressive or steroid therapy than in untreated patients. In our analysis we have considered all patients on steroid therapy as treated (over $5 \mathrm{mg}$ of prednisone/day) because statistically significant differences were found. 
We also utilized PET to detect therapy response in 19 patients affected by LVV. These patients were evaluated at baseline and at a different time point of follow up (when clinical relapse was evident or after steroid therapy reduction or withdrawal). Even if the number of patients was low for statistical analysis we could see a good correlation between positivity of PET and clinical symptoms.

\section{Discussion}

The diagnosis of large vessel vasculitis (LVV) is often difficult, and it usually requires time, money and discomfort for the patient. The diagnosis of small vessel vasculitis is still histological.

As reported by Hooisma (2012), the diagnostic standard method for LVV, especially in giant cell arteritis (GCA), is a temporal artery biopsy, but the test results can be false negative in 15-70\%, which may delay the diagnosis; in Takayasu's arteritis (TA), which affects the aorta and its main branches, as well as the coronary and pulmonary arteries, routine histological examination is not suitable.

Several methods such as Color Doppler Sonography (CDS), Magnetic Resonance (MR) and Computed Tomography (CT) have been proposed to evaluate LVV. As reported by Pipitone et al. (2008) these tools (CDS, MRI, CT) revealed their usefulness in detecting early vasculitic lesions (mainly presented as an alteration of vessel lumen), while only angiography was able to detect delayed effects of LVV (e.g. aneurism or vascular stenosis).

Besson and coworkers (2011) reported in their meta-analysis that diagnostic performance of FDG-PET in giant cell arteritis provided sensitivity and specificity of $80 \%$ and $89 \%$, respectively.

In their study, Ergul (2011) and Hooisma (2012) highlighted the utility of PET in early diagnosis of LVV and in discovery of occult inflammatory or neoplastic disorders.
In our study, the sensitivity and specificity of PET are high, with lower risk to patients with respect to other diagnostic methods (such as angiography or CT/MR angiography). As previously reported by Fuchs et al., ${ }^{18}$ F-FDG PET/CT increases the overall diagnostic accuracy and has an impact on the medical management in a significant proportion of patients, but it is unlikely to replace biopsy procedures in the near future. PET-CT without contrast allows evaluating the extension of vascular involvement; however, it cannot accurately analyze the vascular wall. The intensity of vascular inflammation, moreover, can help in the differential diagnosis and it can be a useful tool for monitoring response to therapy.

As described by Kumar et al (2012)., the correlation between the inflammatory status and FDG uptake also allows to use PET in follow up, for evaluating response to LVV therapy, and to detect possible flares before complications arise.

In a prospective study by Blockmans et al. (2006) involving 35 patients (CAO) with GCA, a significant decrease in vascular FDG uptake at 3 months indicated a potential future response to treatment.

As with previous reports, we confirm the lower diagnostic accuracy in patients on immunosuppressive therapy. We chose a cut off of $5 \mathrm{mg} /$ day of prednisone for a week because this dose does not seem to interfere with SUV, but it is often impractical to not treat a severe vasculitis before obtaining a PET scan.

Even our data, in agreement with what is reported in the literature, do not allow to express an opinion on a possible involvement of cerebral vessels given the high uptake of FDG in brain tissue.

Qui Lin et al. (2012) reviewed the literature on the use of PET in the diagnosis of FUO, and it was found that PET's mean sensitivity was $80-90 \%$ and specificity was 88-90\%. FDG PET scans were examined in various cases. A definitive diagnosis was made in $50 \%$ of cases and $20 \%$ of cases were related to inflammatory processes of 
the great vessels. Therefore, Yang et al. (2012) confirmed the superiority of PET among the various diagnostic techniques used in the diagnosis of FUO, with an excellent cost benefit profile, the possibility of reducing the number of surveys and the duration of hospitalization.

In our study, the use of PET in patients with FUO has allowed us to make a diagnosis in $80 \%$ of cases, of which $70 \%$ was a vasculitis.

\section{Conclusion}

${ }^{18} \mathrm{~F}-\mathrm{FDG}$ PET/CT (PET) is a sensitive and specific imaging tool for large vessel vasculitis (LVV) and FUO; it increases the overall diagnostic accuracy and has an impact on the clinical management in a significant proportion of patients for several reasons. FU0 might be caused by a vasculitis. PET is a one-shot diagnostic method (cost saving) as reported by Becerra et al. (2012). Its diagnostic accuracy precludes the need for arterial biopsy, and instead an easy semiquantitative analysis can be used for the right diagnosis of vasculitis. Finally, PET is a good tool for the diagnosis of LVV, but also for therapy monitoring and follow up.

However, since this method still encounters some resistance to be used as the gold standard in the diagnostic guidelines of FUO, further studies are needed to confirm the specificity, accuracy and sensitivity as well as the cost benefit analysis in the diagnosis of vasculitis.

\section{Disclosures}

None

\section{References}

1. Basu, N., Watts, R., Bajema, I., Baslund, B., Bley, T., Boers, M., et al. (2010), 'EULAR points to consider in the development of classification and diagnostic criteria in systemic vasculitis,' Annals of the Rheumatic Diseases, 69,1744-50.

2. Becerra Nakayo, EM., García Vicente, AM., Soriano Castrejón, AM., Mendoza
Narváez, JA., Talavera Rubio, MP., Poblete García, VM. and Cordero García, JM. (2012), 'Analysis of cost-effectiveness in the diagnosis of fever of unknown origin and the role of (18)F-FDG PET-CT: a proposal of diagnostic algorithm,' Revista Espaňola de Medicina Nuclear e Imagen Molecular, Jul-Aug, 31(4),178-86.

3. Besson, FL., Parienti, JJ., Bienvenu, B., Prior, JO., Costo, S., Bouvard, G. and Agostini, D. (2011), ' Diangnostic performance of 18F-fluorodeoxyglucose positron emission tomography in giant cell arteritis: a systemic review and metaanalysis,' European Journal of Nuclear Medicine and Molecular Imaging, Sept. 38(9), 1764-72.

4. Blockmans, D., de Ceuninck, L., Vanderschueren, S., et al. (2006), 'Repetitive 18F-fluorodeoxyglucose positronemission tomography in giant cell arteritis: a prospective study of 35 patients,' Arthritis and Rheumatology, 55, 131-7.

5. Cao, Q. and Chen, W. (2012), ' FDG PET Imaging of Large-Vessel Vasculitis,' PET Clinics, 7, 227-232.

6. Cheng, Y., Lv, N., Wang, Z., Chen, B. and Dang, A. (2013), '18FDG PET in assessing disease activity in takayasu arteritis: a meta-analysis,' Clinical and Experimental Rheumatology, 31(1 Suppl 75), S22-7.

7. Chow, A. and Robinson, JL. (2011), 'Fever of unknown origin in children: a systematic review,' World Journal of Pediatrics, Feb, 7(1), 5-10.

8. Ergül, N. and Cermik, TF. (2011), 'FDGPET or PET/CT in fever of unknown origin: the diagnostic role of underlying primary disease,' International Journal of Molecular Imaging, 2011, 318051.

9. Fuchs, M., Briel, M., Daikeler, T., Walker, UA., Rasch, H., Berg, S., Ng, Q., Raatz, H., Jayne, D., Kötter, I., Blockmans, D., Cid, MC., Prieto-González, S., Lamprecht, P., Salvarani, C., Karageorgaki, Z., Watts, R., Luqmani, R., Müller-Brand, J., Tyndall, A. and Walter, MA. (2012), 'The 
impact of 18F-FDG PET on the management of patients with suspected large vessel vasculitis,' European Journal of Nuclear Medicine and Molecular Imaging, 39, 344-35.

10. Gaeta, GB., Fusco, FM. and Nardiello, S. (2006), 'Fever of unknown origin: a systematic review of the literature for 1995-2004,' Nuclear Medicine Communications, 27(3), 205-11.

11. Hooisma, GA., Balink, H., Houtman, PM., Slart, RHJA. and Lensen, KDF. (2012), 'Parameters related to a positive test result for FDG PET(/CT) for large vessel vasculitis: a multicenter retrospective study,' Clinical Rheumatology, 31, 861871.

12. Jennette, C., Falk, RJ., Bacon, PA., Basu, N., Cid, MC., Ferrario, F., Flores-Suarez, LF., Gross, WL., Guillevin, L., et al (2013), '2012 Revised International Chapel Hill Consensus Conference Nomenclature of Vasculitides,' Arthritis and Rheumatology, 65(65), 1-11.

13. Kumar, R., Karunanithi, S., Zhuang, H., Alavi, A. (2012), 'Assessment of Therapy Response by FDG PET in Infection and Inflammation,' PET Clinics, 7, 233-243.

14. Meller, J., Sahlmann, CO. and Scheel, AK. (2007), '18F-FDG PET and PET/CT in fever of unknown origin,' Journal of Nuclear Medicine, 48(1), 35-45.

15. Mukhtyar, C., Guillevin, L., Cid, MC., Dasgupta, B., de Groot, K., Gross, W., et al. (2009), 'EULAR recommendations for the management of large vessel vasculitis,' Annals of the Rheumatic Diseases, 68, 31823.

16. Papathanasiou, ND., Du, Y., Menezes, LJ., Almuhaideb, A., Shastry, M., Beynon, H. and Bomanji, JB. (2012). '18FFludeoxyglucose PET/CT in the evaluation of large-vessel vasculitis: Diagnostic performance and correlation with clinical and laboratory parameters,' British Journal of Radiology, 85, 1014 (e188e194).
17. Pipitone, N., Versari, A. and Salvarani, C. (2008), 'Role of imaging studies in the diagnosis and follow-up of large-vessel vasculitis: an update,' Rheumatology, 47, 403-408.

18. Qiu, L. and Chen, Y. (2012), 'The role of 18F-FDG PET or PET/CT in the detection of fever of unknown origin,' European Journal of Radiology, 81, 3524- 3529.

19. Roth, AR. and Basello, GM. (2003), 'Approach to the adult patient with fever of unknown origin,' American Family Physician, 68(11), 2223-8.

20. Schmidt, WA. (2013), 'Imaging in vasculitis,' Best practice \& research clinical rheumatology, 27, 107-118.

21. Sheng, JF., Sheng, ZK., Shen, XM., Bi, S., Li, JJ., Sheng, GP., Yu, HY., Huang, HJ., Liu, J., Xiang, DR., Dong, MJ., Zhao, K. and Li, LJ. (2011), 'Diagnostic value of fluorine-18 fluorodeoxyglucose positron emission tomography/computed tomography in patients with fever of unknown origin,' European Journal of Internal Medicine, 22(1), 112-6.

22. Talarico, R., Baldini, C., Della Rossa, A., Stagnaro, C., Ferrari, C., Luciano, N. and Bombardieri, S. (2012), 'Large- and smallvessel vasculitis: a critical digest of the 2010-2011 literature,' Clinical and Experimental Rheumatology, 30(Suppl. 70), S130-S138.

23. Tezuka, D., Go Haraguchi, G., Ishihara, T., Ohigashi, H., Inagaki, H., Suzuki, J., Hirao, K. and Isobe, M. (2012), 'Role of FDG PET-CT in Takayasu Arteritis Sensitive Detection of Recurrences,' Journal of the American College of Cardiology, 5(4), 422-9.

24. Watts, R., Al-Taiar, A., Mooney, J., et al. (2009), 'The epidemiology of Takayasu arteritis in the UK,' Rheumatology(Oxford), 48(8), 1008-11.

25. Yang, J., Zhuang, H. and Servaes, S. (2012), 'Fever of Unknown Origin: The Roles of FDG PET or PET/CT,' PET Clinics, 7, 181-189. 